Abstract

\title{
Disrupting Androgen Receptor Functionality with Xanthones from the Mangosteen (Garcinia mangostana) Fruit ${ }^{+}$
}

Jeremy J. Johnson

College of Pharmacy, University of Illinois at Chicago, Chicago, IL 60612, USA; jjjohn@uic.edu

+ Presented at the 3rd International conference on Natural Products for Cancer Prevention and Therapy, Kayseri, Turkey, 18-20 December 2019.

Published: 3 March 2020

\begin{abstract}
The Southeast Asian mangosteen (Garcinia mangostana) contains a class of phytochemicals known as xanthones that possess extensive biological activity. Applications of xanthones, including the most prominent, alpha-mangostin, have been shown to possess anti-cancer, anti-oxidant, and anti-proliferation properties. To confirm the anti-cancer activity of xanthones we have evaluated 9 xanthones for decreasing cellular proliferation of cancer cells. These xanthones include alphamangostin, gartanin, 9-hydroxycalabaxanthone, garcinone C, garcinone D, and others. Using this approach, we have focused on understanding the ability of xanthones to disrupt androgen receptor in prostate cancer cells with a combination of cell free and cell-based assays. In addition, we have performed pharmacokinetic studies in mice with alpha-mangostin to characterize the optimal dosing strategy. Taken together, we have identified individual xanthones as capable of disrupting kinases, including CDK4, using cell free biochemical models and cell-based animal models. These results have been further validated in an in vivo xenograft model. Taken together, we have begun to describe the anti-cancer potential of xanthones for prostate cancer.
\end{abstract}

Keywords: mangosteen; Garcinia mangostana; mangostin; prostate cancer; androgen receptor

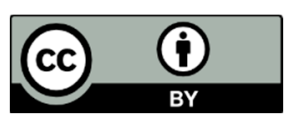

(C) 2020 by the authors. Licensee MDPI, Basel, Switzerland. This article is an open access article distributed under the terms and conditions of the Creative Commons Attribution (CC BY) license (http://creativecommons.org/licenses/by/4.0/). 